\title{
Anisotropic interactions of a single spin and dark-spin spectroscopy in diamond
}

\author{
R. J. EPSTEIN, F. M. MENDOZA, Y. K. KATO AND D. D. AWSCHALOM* \\ Center for Spintronics and Quantum Computation, University of California, Santa Barbara, California 93106, USA \\ *e-mail: awsch@physics.ucsb.edu
}

E xperiments on single nitrogen-vacancy $(\mathrm{N}-\mathrm{V})$ centres in diamond, which include electron spin resonance $^{1}$, Rabi oscillations $^{2}$, single-shot spin readout ${ }^{3}$ and two-qubit operations with a nearby ${ }^{13} \mathrm{C}$ nuclear $\operatorname{spin}^{4}$, show the potential of this spin system for solid-state quantum information processing. Moreover, $\mathrm{N}-\mathrm{V}$ centre ensembles can have spin-coherence times exceeding $50 \mu \mathrm{s}$ at room temperature ${ }^{5}$. We have developed an angle-resolved magneto-photoluminescence microscope apparatus to investigate the anisotropic electron-spin interactions of single $\mathrm{N}-\mathrm{V}$ centres at room temperature. We observe negative peaks in the photoluminescence as a function of both magnetic-field magnitude and angle that are explained by coherent spin precession and anisotropic relaxation at spin-level anti-crossings. In addition, precise field alignment unmasks the resonant coupling to neighbouring 'dark' nitrogen spins, otherwise undetected by photoluminescence. These results demonstrate the capability of our spectroscopic technique for measuring small numbers of dark spins by means of a single bright spin under ambient conditions.

The $\mathrm{N}-\mathrm{V}$ defect pair, with trigonal symmetry ${ }^{6}$, has an anisotropic electron-spin hamiltonian owing to spin-spin and spin-orbit interactions ${ }^{7}$. Consequently, the degrees of spin-level mixing and coupling to nearby impurity spins are very sensitive to the orientation of an applied magnetic field, which has not been controlled in experiments on single $\mathrm{N}-\mathrm{V}$ centres. Figure 1a depicts the atomic structure and relevant energy levels of the (negatively charged) $\mathrm{N}-\mathrm{V}$ centre. The triplet $\left({ }^{3} \mathrm{~A}\right)$ ground state $^{8-10}$ has a zero-field splitting between the $\left|m_{\mathrm{S}}=0\right\rangle$ and $\left|m_{\mathrm{S}}= \pm 1\right\rangle$ spin sublevels, where $m_{\mathrm{S}}$ is the quantum number of the spin sublevel, quantized along the $\mathrm{N}-\mathrm{V}$ symmetry axis, a $\langle 111\rangle$ crystal axis ${ }^{11}$. Zero-field spin splittings have also been measured in the triplet $\left({ }^{3} \mathrm{E}\right)$ excited state but there is no consensus on the arrangement of sublevels ${ }^{12-15}$. Linearly polarized optical excitation of the ${ }^{3} \mathrm{~A} \rightarrow{ }^{3} \mathrm{E}$ transition preferentially pumps the spin system into the $|0\rangle$ ground-state sublevel ${ }^{16}$. In addition, the average photonemission rate is substantially smaller for transitions involving the $| \pm 1\rangle$ levels than for the $|0\rangle$ level $^{3}$, which enables the spin state to be determined by the photoluminescence intensity $I_{\mathrm{PL}}$. Both of the latter two effects have been attributed to spin-dependent intersystem crossing to the singlet $\left({ }^{1} \mathrm{~A}\right)$ level ${ }^{11,17}$.
The single-crystal samples investigated here are commercially available (Sumitomo Electric Industries) high-temperature highpressure diamond with two polished parallel (100) surfaces and nominal dimensions of $1.4 \mathrm{~mm} \times 1.4 \mathrm{~mm} \times 1.0 \mathrm{~mm}$. They contain nitrogen impurities with densities of $10^{19}-10^{20} \mathrm{~cm}^{-3}$, measured by ultraviolet absorption ${ }^{18}$. N-V centres are naturally present with substantially lower densities ranging from $10^{10}$ to $10^{13} \mathrm{~cm}^{-3}$. For ensemble measurements, samples are irradiated with $1.7-\mathrm{MeV}$ electrons with a dose of $5 \times 10^{17} \mathrm{~cm}^{-3}$ and subsequently annealed at $900{ }^{\circ} \mathrm{C}$ for $2 \mathrm{~h}$ to increase the $\mathrm{N}-\mathrm{V}$ centre concentration ${ }^{6}$.

The phonon-broadened ${ }^{3} \mathrm{E} \rightarrow{ }^{3} \mathrm{~A}$ transition of the $\mathrm{N}-\mathrm{V}$ centre is detected by means of non-resonant photoluminescence microscopy (see Methods). For example, Fig. 1b is a spatial image of the spectrally integrated photoluminescence from a diamond sample with the laser focused roughly $1 \mu \mathrm{m}$ below the surface. Multiple resolution-limited features are observed in the $20 \mu \mathrm{m} \times 20 \mu \mathrm{m}$ field. In order to determine that a given feature is due to a single emitter, a histogram is plotted of the time $\tau$ between consecutive photon detection events using a Hanbury Brown and Twiss detection geometry ${ }^{19}$, yielding the experimental intensity correlation function $g^{(2)}(\tau)$. Figure 1c shows the data from a colour centre labelled NV1. The value of $g^{(2)}(0)$ is below 0.5 , proving that $\mathrm{NV} 1$ is a single-colour centre ${ }^{20}$. In addition, the rise of $g^{(2)}(\tau)$ above unity with increasing $|\tau|$ is indicative of intersystem crossing to the ${ }^{1} \mathrm{~A}$ metastable state ${ }^{20,21}$.

These single-photon emitters are further characterized by optically detected electron spin resonance (ESR; see Methods). Figure 1d contains data from NV1 with zero applied magnetic field. In this case, the $| \pm 1\rangle$ ground-state levels are degenerate so that only one resonance $\left(m_{\mathrm{S}}=0 \rightarrow \pm 1\right)$ is observed at $2.87 \mathrm{GHz}$, the characteristic zero-field splitting of $\mathrm{N}-\mathrm{V}$ centres ${ }^{11}$. The data are fit with a lorentzian of $11 \mathrm{MHz}$ full-width at half-maximum (solid line), which is comparable to reported values in similar diamond samples ${ }^{1}$.

Strain-dependent optical measurements on ensembles of N-V centres have indicated that electric dipole transitions are allowed for dipoles in the plane perpendicular to the symmetry axis ${ }^{6}$. Figure $2 \mathrm{a}$ depicts an $\mathrm{N}-\mathrm{V}$ centre with transition dipoles $\mathbf{X} \|[\overline{1} \overline{1} 2]$ and $\mathbf{Y} \|[1 \overline{1} 0]$ in a (111) plane. The excitation is along [001] for all measurements, therefore $I_{\mathrm{PL}}$ depends on the laser polarization angle 
a

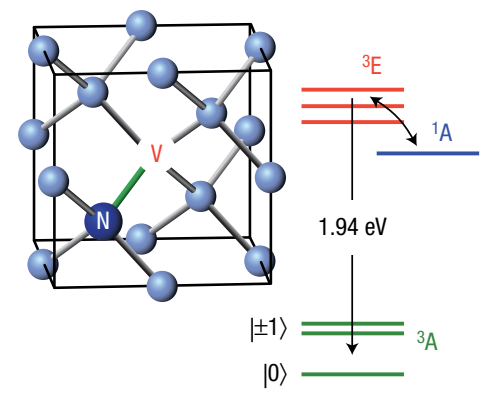

G

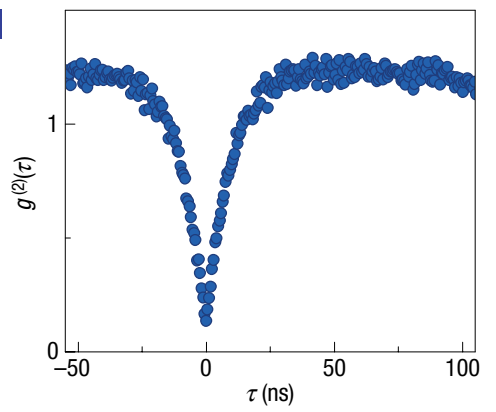

b

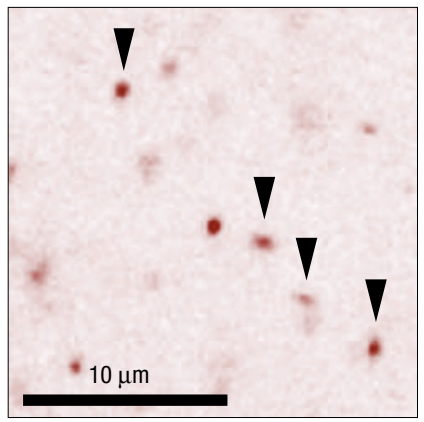

d

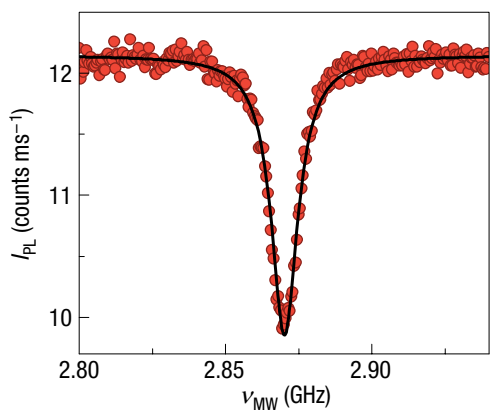

Figure 1 Characterization of single $\mathbf{N}-\mathbf{V}$ centres. a, The atomic structure and relevant energy levels. The green 'bond' depicts the N-V symmetry axis. b, The spatial image of $I_{\mathrm{PL}}$ on a linear colour scale, showing the emission from several $\mathrm{N}-\mathrm{V}$ centres; NV1-NV4 (left to right) are marked. c, The intensity correlation function $g^{(2)}(\tau)$ versus $\tau$ for NV1, indicating single photon emission. d, Optically detected ESR for NV1. $I_{\mathrm{PL}}$ versus microwave frequency $v_{\mathrm{MW}}$ (circles) with a lorentzian fit (solid line). The laser power is $1 \mathrm{~mW}$ for $\mathbf{b}$ and $370 \mu \mathrm{W}$ for $\mathbf{c}$ and $\mathbf{d}$.
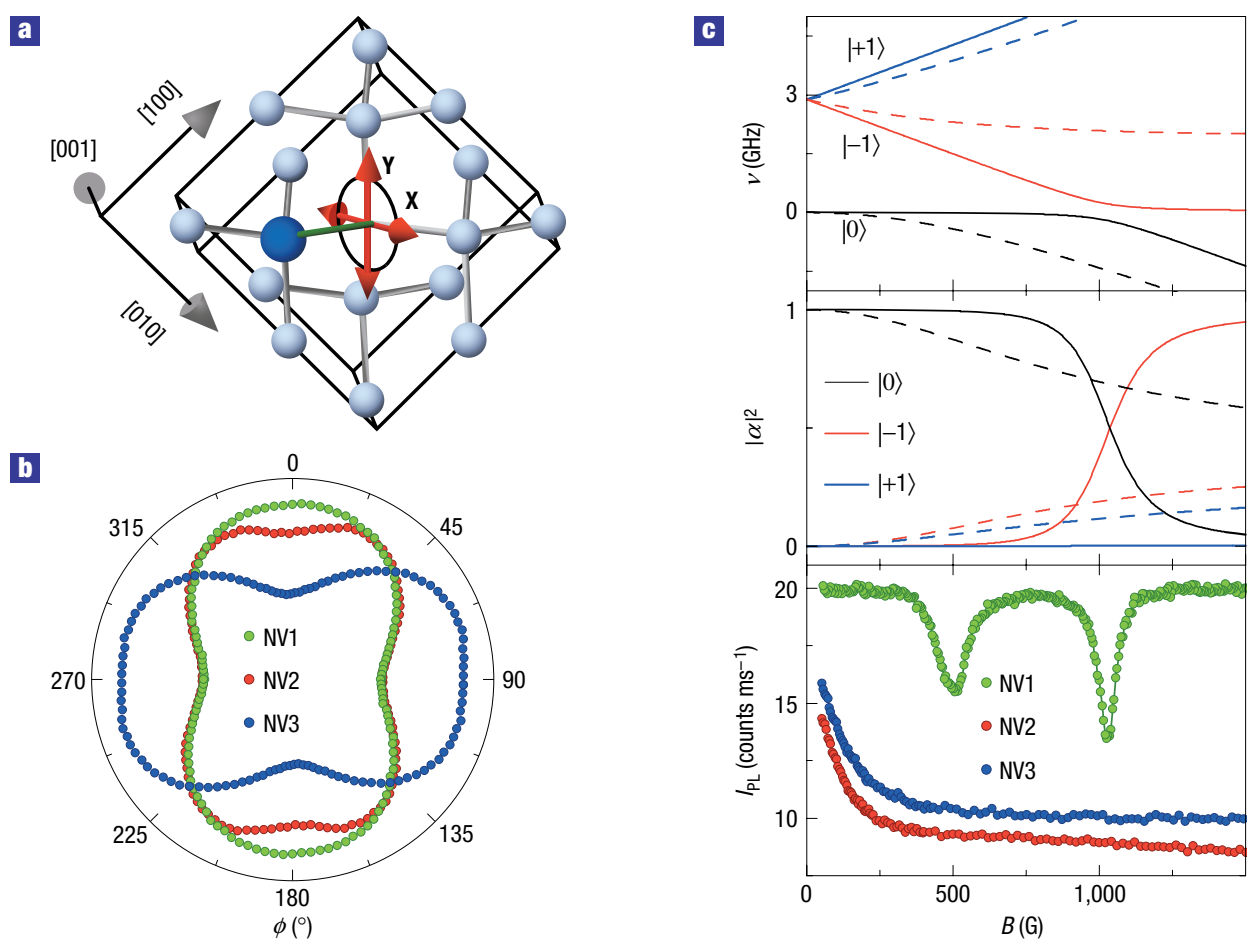

Figure 2 Polarization and magnetic-field anisotropies. a, Measurement geometry indicating transition dipoles $\mathbf{X}$ and $\mathbf{Y}$. b, Normalized $l_{\mathrm{PL}}$ (radial axis) versus the laser polarization angle $\phi$ for NV1, NV2 and NV3. Polarization along [110] corresponds to $\phi=0^{\circ}$ and data at $\phi$ and $\phi+180^{\circ}$ are identical. c, Upper panel: calculated ground-state spin splitting $v$ as a function of $B$ for $\theta=6^{\circ}$ (solid lines) and $54.7^{\circ}$ (dashed lines). Middle panel: overlap $|\alpha|^{2}$ of each spin level with $|0\rangle_{z}$ at the same two angles. Lower panel: $I_{\mathrm{PL}}$ versus $B$ for NV1, NV2 and NV3, with $\sim 1^{\circ}$ angle between $\mathbf{B}$ and [111]. The laser power is $370 \mu \mathrm{W}$ for $\mathbf{b}$ and $1 \mathrm{~mW}$ for $\mathbf{c}$. 

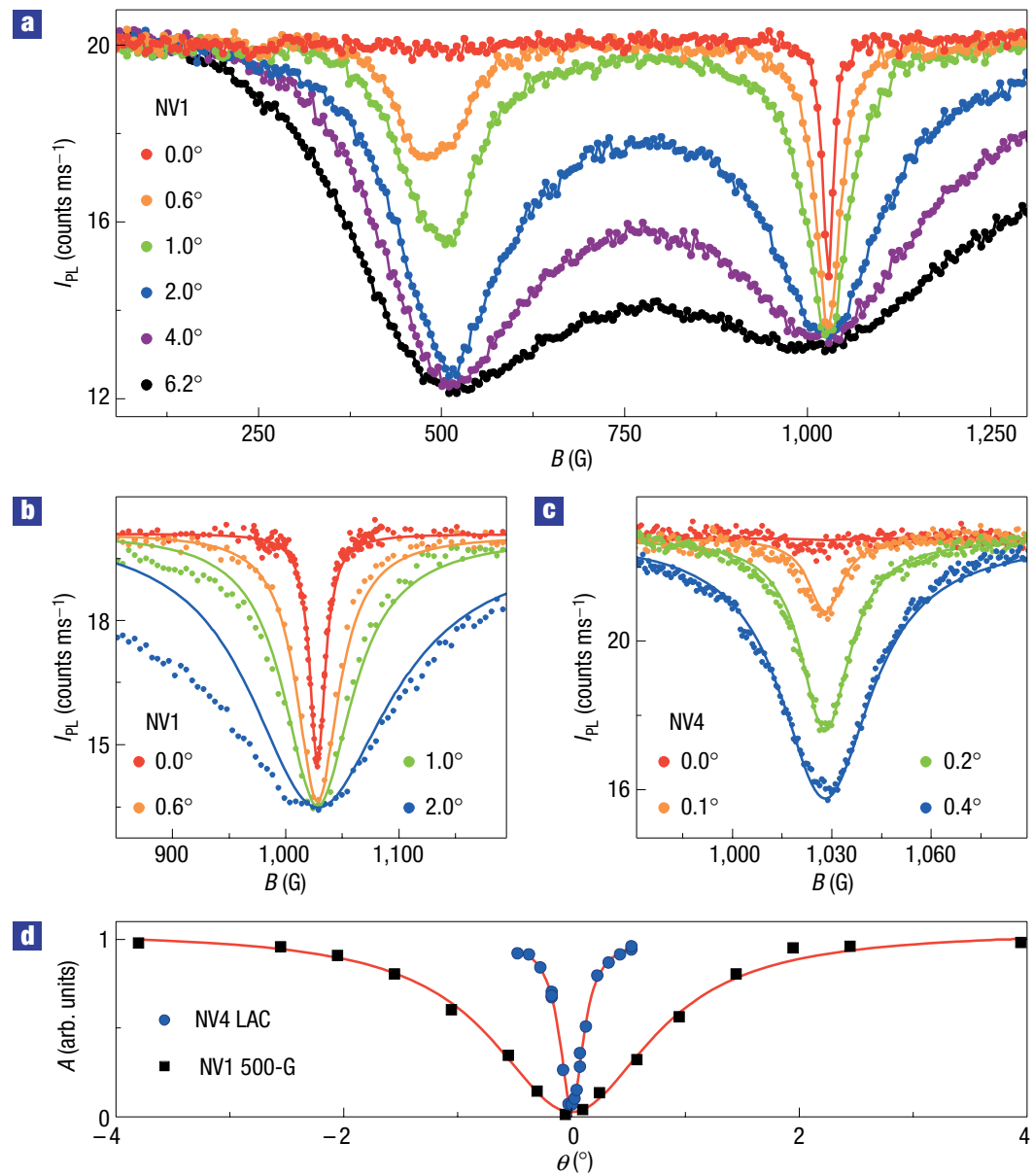

Figure 3 Controlled level mixing by means of magnetic-field alignment. a, $l_{\mathrm{PL}}$ versus $B$ for NV1 at specified magnetic-field angles $\theta$. b, Zoom of data in a (points) with fits (lines) to model described in the text. Data for $\theta=0^{\circ}$ are taken with smaller field steps and fit with an angle of $0.2^{\circ}$ to account for residual spin mixing (see the text). c, $I_{\mathrm{PL}}$ versus $B$ for NV4 (points) and fits (lines) at specified field angles. d, Normalized amplitudes $A$ (points) of LAC and 500-G peaks versus $\theta$ with fits (lines). Laser power is 1 mW for NV1 and $2.9 \mathrm{~mW}$ for NV4.

$\phi$ owing to unequal excitation of the two dipoles. The dependence of $I_{\mathrm{PL}}$ on $\phi$ is measured for $20 \mathrm{~N}-\mathrm{V}$ centres and shows either vertical or horizontal lobes, as exemplified for three N-V centres in Fig. 2 b. The measured anisotropies, defined as the ratio $I_{\mathrm{PL}}\left(\phi=0^{\circ}\right) / I_{\mathrm{PL}}$ $\left(\phi=90^{\circ}\right)$, are roughly $2: 1$ and $1: 2$ on average; a simple anisotropy calculation gives $3: 1,3: 1,1: 3$ and $1: 3$ for the $\mathrm{N}-\mathrm{V}$ symmetry axis

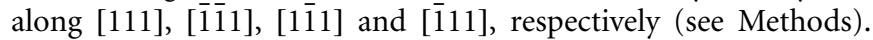
The measured data are modified by the presence of polarized background photoluminescence (subtracted from the data) and a small dip, most visible for NV2 at $\phi=0^{\circ}$, which varies in depth from centre to centre. Nevertheless, comparison of the measured and calculated anisotropies enables the number of possible orientations of a given centre to be reduced from four to two.

The double degeneracy of the polarization anisotropy is lifted by application of a magnetic field $\mathbf{B}$. The upper panel of Fig. 2c shows the ground-state spin levels as a function of $B$ (the amplitude of $\mathbf{B}$ ) calculated using the hamiltonian ${ }^{7}$ $H=g \mu_{\mathrm{B}} \mathbf{B} \cdot \mathbf{S}+D\left(S_{z}^{2}-S(S+1) / 3\right)$, where $\mu_{\mathrm{B}}$ is the Bohr magneton, $g=2.00$ is the electron $g$-factor, $S$ is the total electron spin with magnitude $S=1$ and $D=2.88 \mathrm{GHz}$ is the ground-state spin splitting. A level anti-crossing (LAC) near 1,000 G is predicted for an $\mathrm{N}-\mathrm{V}$ centre with symmetry axis at a small angle to $\mathbf{B}\left(6^{\circ}\right.$; solid lines), but not for a large angle $\left(54.7^{\circ}\right.$; dashed lines). The middle panel shows $|\alpha|^{2}$, the overlap of $|0\rangle_{z}$ with each spin level,
$\left|m_{\mathrm{S}}\right\rangle=\alpha|0\rangle_{z}+\beta|-1\rangle_{z}+\gamma|+1\rangle_{z}$, where $|\beta|^{2}$ and $|\gamma|^{2}$ are the other respective overlaps and the subscript $z$ denotes the [111] basis; the evolution of $|\alpha|^{2}$ with $B$, calculated for both angles, illustrates the mixing of spin states and will be relevant in modelling the data. The lower panel is a plot of $I_{\mathrm{PL}}$ as a function of $B$, with an angle of $\sim 1^{\circ}$ between $\mathbf{B}$ and [111], for the same three N-V centres as in Fig. $2 b$. Whereas NV1 and NV2 have similar polarization dependences, their field dependences show that the symmetry axis of NV1 (NV2) is (non-)parallel to B. For NV1, the negative peak at $\sim 1,000 \mathrm{G}$ coincides with the calculated LAC. In addition, NV1 shows a peak at $\sim 500 \mathrm{G}$, the origin of which is less clear.

In order to investigate these peaks further, $I_{\mathrm{PL}}$ is measured as a function of $B$ with $\mathbf{B}$ at a series of angles $\theta$ in the (110) plane; a selection of such data from NV1 is shown in Fig. 3a. As B approaches the [111] direction $\left(\theta \rightarrow 0^{\circ}\right)$, the LAC peak narrows, as expected from ensemble measurements ${ }^{22,23}$. At sufficiently small angles, however, the LAC peak amplitude decreases for NV1 (Fig. 3b) or even vanishes for some centres, such as NV4 (Fig. 3c). This is in contrast to the ensemble measurements ${ }^{22,23}$ that showed a maximum in the LAC peak amplitude at $\theta=0^{\circ}$. According to the hamiltonian above, however, spin mixing should vanish at $\theta=0^{\circ}$. The presence of residual spin mixing at $\theta=0^{\circ}$ has been attributed to strain and nuclear interactions ${ }^{24}$, which vary from centre to centre. 

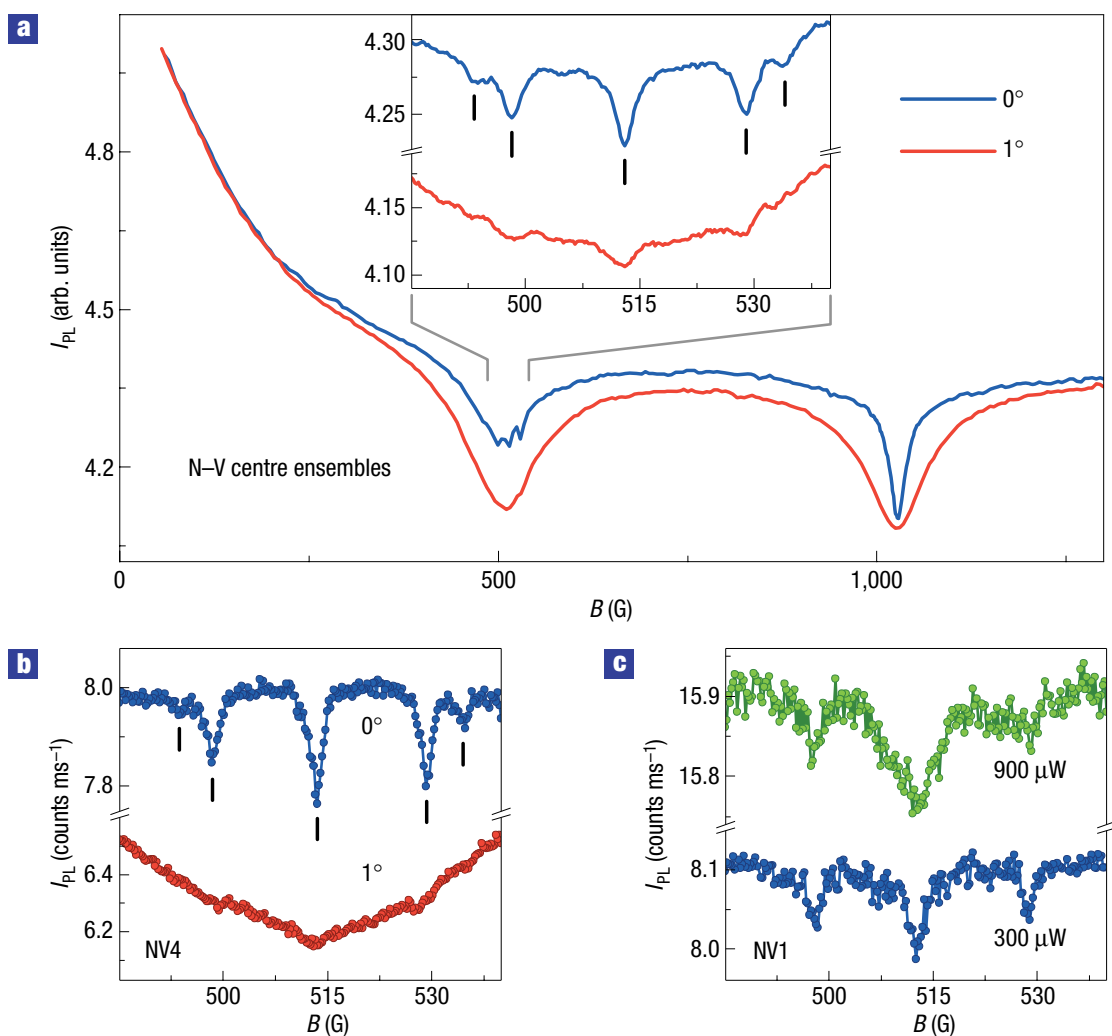

Figure 4 Resonant coupling of a single spin to neighbouring spins. a, $l_{\mathrm{PL}}$ versus $B$ for an ensemble of $\mathrm{N}-\mathrm{V}$ centres at two field angles. Inset: higher-resolution scans around $500 \mathrm{G}$ showing the nitrogen hyperfine structure. $\mathbf{b}, \mathrm{A}$ single $\mathrm{N}-\mathrm{V}$ centre (NV4) coupling to several nitrogen centres. $I_{\mathrm{PL}}$ versus $B$ at two indicated field angles with $300 \mu \mathrm{W}$ laser power. c, $I_{\mathrm{PL}}$ versus $B$ at two indicated laser powers for NV1, showing broadening of nitrogen peaks with increased power. For a (inset), b and $\mathbf{c}$, a small linear background is subtracted from the data.

The LAC can be modelled for small values of $\theta$ by considering $|0\rangle$ and $|-1\rangle$ as pseudo-spin- $1 / 2$ states. With preferential population of $|0\rangle_{z}$, the $|+1\rangle$ level is ignored because it has a negligible overlap with $|0\rangle_{2}$ (see Fig. 2c, middle panel). We then have an effective hamiltonian $H=g \mu_{\mathrm{B}}\left(\mathbf{B}-\mathbf{B}_{0}\right) \cdot \mathbf{s}$, where $\mathbf{B}_{0}$ accounts for the zero-field spin splitting and $\mathbf{s}$ is the pseudo-spin operator. The Bloch equations for $\mathbf{B}$ in the (110) plane are taken to be

$$
\begin{gathered}
\frac{\mathrm{d} s_{x}}{\mathrm{~d} t}=-\Omega_{z} s_{y}-\frac{s_{x}}{T_{2}}, \\
\frac{\mathrm{d} s_{y}}{\mathrm{~d} t}=\Omega_{z} s_{x}-\Omega_{x} s_{z}-\frac{s_{y}}{T_{2}}, \\
\frac{\mathrm{d} s_{z}}{\mathrm{~d} t}=\Omega_{x} s_{y}-\frac{s_{z}}{T_{1}}+\Gamma,
\end{gathered}
$$

where $\hbar \boldsymbol{\Omega}=g \mu_{\mathrm{B}}\left(\mathbf{B}-\mathbf{B}_{0}\right), \hbar$ is the reduced Planck constant, $\boldsymbol{\Omega}=\left(\Omega_{x}, \Omega_{y}, \Omega_{z}\right)$ is the Larmor precession vector, $x \|[\overline{1} \overline{1} 2]$, $y\left\|[1 \overline{1} 0], \mathbf{B}_{0}\right\| z \|[111], \Gamma$ is the rate of optical spin orientation along $z$, and $T_{1}$ and $T_{2}$ are effective spin relaxation times that depend on $\Gamma$ (see Methods). In this model, the spin relaxation is anisotropic in that $T_{1}$ and $T_{2}$ are fixed relative to the crystal axes rather than the magnetic field. The steady-state solution for $s_{z}$ is

$$
s_{z}=\frac{T_{1} \Gamma\left(1+\Omega_{z}^{2} T_{2}^{2}\right)}{1+\Omega_{x}^{2} T_{1} T_{2}+\Omega_{z}^{2} T_{2}^{2}} .
$$

We then take $I_{\mathrm{PL}}=a n_{0}+b n_{-1}=a\left(1 / 2+s_{z}\right)+b\left(1 / 2-s_{z}\right)$, where $a$ and $b\left(n_{0}\right.$ and $\left.n_{-1}\right)$ are the photoluminescence rates (occupation probabilities) for the $|0\rangle_{z}$ and $|-1\rangle_{z}$ levels, respectively. This simple model describes the experimental data for a wide range of angles and magnetic fields, where the same fit parameters are used for all angles (Fig. 3b,c lines). The fits yield $T_{1}=64 \mathrm{~ns}$ and $T_{2}=11 \mathrm{~ns}$ for NV4 using a laser power of $2.9 \mathrm{~mW}$ (Fig. 3c) and $T_{1}=130 \mathrm{~ns}$ and $T_{2}=23 \mathrm{~ns}$ for a power of $900 \mu \mathrm{W}$ (not shown). The results indicate that the laser introduces substantial anisotropic spin relaxation by means of excitation out of the ground-state manifold. This source of decoherence can be mitigated through pulsed excitation ${ }^{4}$.

The 500-G peak evolves similarly to the LAC peak but over a broader angular range. It has nearly the same amplitude and width at a given field angle for all $\mathrm{N}-\mathrm{V}$ centres investigated with suitable orientation. In addition, the peak has been observed in all four samples measured. The evolution of the 500-G peak with $\theta$ and B can be reasonably accounted for in the model by postulating an LAC in the excited state, as is expected to occur at some field owing to the presence of zero-field spin splittings $\mathrm{s}^{12-14}$. Figure $3 \mathrm{~d}$ shows the normalized amplitudes $A$ of the $500-G$ and LAC (1,000-G) peaks as a function of $\theta$. Fits of the model to this data (red lines) and to the field scans (fits not shown) yield $T_{1}=36 \mathrm{~ns}$ and $T_{2}=1.8 \mathrm{~ns}$ for an excited-state LAC at $\sim 500 \mathrm{G}$.

Similar data are taken on ensembles of N-V centres in Fig. $4 \mathrm{a}$, where the $500-\mathrm{G}$ peak is found to reduce in amplitude by $\sim 50 \%$ at $\theta=0^{\circ}$. In addition, higher-resolution field scans around $500 \mathrm{G}$ (Fig. 4a, inset) show a quintuplet of peaks characteristic of the hyperfine structure of substitutional nitrogen $\left(\mathrm{N}_{\mathrm{S}}\right)$ centres $^{25}$. These peaks appear when the magnetic field tunes the electron spin splitting of $\mathrm{N}-\mathrm{V}$ centres into resonance with the many surrounding 
$\mathrm{N}_{\mathrm{S}}$ centres, resulting in enhanced cross-relaxation by means of the magnetic dipole interaction ${ }^{26-28}$. Notably, the $\mathrm{N}_{S}$ peak amplitudes diminish as $|\theta|$ increases, suggesting that the $500-\mathrm{G}$ peak is associated with a decrease in spin polarization, like the LAC peak.

The above results point to a regime $\left(|\theta|<1^{\circ}\right)$ where the depolarizing effects of the $500-\mathrm{G}$ peak are mitigated, showing the coupling of a single $\mathrm{N}-\mathrm{V}$ centre to its neighbouring $\mathrm{N}_{S}$ spins. Figure $4 \mathrm{~b}$ shows two field scans of $I_{\mathrm{PL}}$ for NV4 at $\theta=0^{\circ}$ and $1^{\circ}$; the $\mathrm{N}_{\mathrm{S}}$ resonances become visible with a sufficiently small angle between $\mathbf{B}$ and [111]. Figure 4c shows similar data for NV1 at $\theta=0^{\circ}$ with powers of 300 and $900 \mu \mathrm{W}$; the $\mathrm{N}_{\mathrm{S}}$ peaks broaden and diminish in relative magnitude at higher power, possibly resulting from ionization of the $\mathrm{N}_{\mathrm{S}}$ centres $^{29}$. The depths of the $\mathrm{N}_{S}$ peaks, differing for NV4 and NV1, are sensitive to the particular spatial distribution of nearby nitrogen spins.

Finally, it is worth noting that the $\mathrm{N}_{\mathrm{S}}$ centres are 'dark' in that they are not directly detected by photoluminescence. By measuring a single $\mathrm{N}-\mathrm{V}$ centre, the number of $\mathrm{N}_{\mathrm{S}}$ spins that can be probed is decreased by orders of magnitude relative to the ensemble measurements. Furthermore, this dark-spin spectroscopy technique is in principle applicable to a variety of paramagnetic defects in diamond. With higher purity samples and single-ion implantation $^{30}$, these results could make possible the long-range coupling of two individually addressable $\mathrm{N}-\mathrm{V}$ centres connected by a chain of dark spins, enabling experimental tests of spin-lattice theories and quantum information processing schemes.

\section{METHODS}

\section{EXPERIMENTAL TECHNIOUES}

The measurement apparatus is based on a confocal microscope with a Hanbury Brown and Twiss detection scheme ${ }^{19}$. A diode-pumped solid-state laser emitting at $532 \mathrm{~nm}$ is linearly polarized and focused onto the sample with a microscope objective of numerical aperture 0.73 and working distance $4.7 \mathrm{~mm}$. The linear polarization is set to any desired angle by changing the retardance of a variable wave plate (fast axis at $45^{\circ}$ to the initially vertical polarization) followed by a quarter-wave plate (fast axis vertical). The laser spot is positioned on the sample in both lateral dimensions with a fast steering mirror. The photoluminescence from the sample is collected by the same microscope objective, passed through a dichroic mirror and a 640 -nm long-pass filter, and sent through a 50/50 beam-splitter to two fibre-coupled silicon avalanche photodiode modules.

For antibunching measurements, the outputs of the detectors are connected to a time-correlated single-photon counting module. The data are normalized by the detector count rates, time bin width and total integration time to yield $g^{(2)}(\tau)$. The data are not corrected for background photoluminescence. For measurements of $\mathrm{N}-\mathrm{V}$ centre ensembles, photoluminescence is detected with a photodiode and lock-in amplifier. In general, the photoluminescence is used as a feedback signal to compensate for thermal drift, enabling a single $\mathrm{N}-\mathrm{V}$ centre to be tracked for several days. The samples are at room temperature for all measurements discussed in this letter.

The static magnetic field $\mathbf{B}$ is applied to the sample with a permanent magnet mounted on a multiaxis stage that allows the distance between the magnet and the sample to be adjusted by a stepper motor, thereby setting $B$ at the sample. In addition, the polar and azimuthal angles of $\mathbf{B}$ are manually adjustable with micrometers while keeping $B$ constant at the sample to better than $1 \%$. For ESR measurements, a $25-\mu \mathrm{m}$-diameter gold wire is connected to a microwave signal generator (with $16 \mathrm{~dB}$ m power output) and placed in close proximity $(\sim 50 \mu \mathrm{m})$ to the laser spot.

\section{POLARIZATION ANISOTROPY CALCULATION}

According to Fermi's golden rule in the electric dipole approximation, the absorption rate is proportional to $|\mathbf{D} \cdot \mathbf{E}|^{2}$, where $\mathbf{D}$ is the dipole matrix element and $\mathbf{E}$ is the excitation electric-field vector. The absorption anisotropy is estimated to be $|\mathbf{Y} \cdot \mathbf{V}|^{2} /|\mathbf{X} \cdot \mathbf{H}|^{2}$, where $\mathbf{X}$ and $\mathbf{Y}$ are the dipoles (see Fig. 2a) and $\mathbf{V} \|[1 \overline{1} 0]$ and $\mathbf{H} \|[110]$ are polarization vectors. A more accurate calculation would account for the dipole radiation pattern, the large numerical aperture of the microscope objective and refraction at the diamond-air boundary.

However, the simple equation above is sufficient to explain both the anisotropy orientations and degeneracies that are measured.

\section{BLOCHEQUATION SIMPLIFICATIONS}

Equation (1) has been simplified by defining effective relaxation times $T_{1}$ and $T_{2}$ that depend on $\Gamma$. Explicitly, $\left(T_{1}\right)^{-1}=\left(T_{z}\right)^{-1}+2 \Gamma$, where $T_{z}$ is the intrinsic relaxation time of $s_{z}$. Likewise, $T_{2}$ has a similar form. As $\Gamma$ is proportional to the laser power, the equation above explains why $T_{1}$ and $T_{2}$ decrease with increasing power, as observed in the experiments.

\section{Received 29 July 2005; accepted 6 September 2005; published 16 October 2005}

\section{References}

1. Gruber, A. et al. Scanning confocal optical microscopy and magnetic resonance on single defect centers. Science 276, 2012-2014 (1997).

2. Jelezko, F., Gaebel, T., Popa, I., Gruber, A. \& Wrachtrup, J. Observation of coherent oscillations in a single electron spin. Phys. Rev. Lett. 92, 076401 (2004).

3. Jelezko, F. et al. Single spin states in a defect center resolved by optical spectroscopy. Appl. Phys. Lett. 81, 2160-2162 (2002)

4. Jelezko, F. et al. Observation of coherent oscillation of a single nuclear spin and realization of a two-qubit conditional quantum gate. Phys. Rev. Lett. 93, 130501 (2004).

5. Kennedy, T. A., Colton, J. S., Butler, J. E., Linares, R. C. \& Doering, P. J. Long coherence times at $300 \mathrm{~K}$ for nitrogen-vacancy center spins in diamond grown by chemical vapor deposition. Appl. Phys. Lett. 83, 4190-4192 (2003).

6. Davies, G. \& Hamer, M. F. Optical studies of the $1.945 \mathrm{eV}$ vibronic band in diamond. Proc. R. Soc. A 348, 285-298 (1976).

7. Pryce, M. H. L. A modified perturbation procedure for a problem in paramagnetism. Proc. Phys. Soc. A 63, 25-29 (1950).

8. Reddy, N. R. S., Manson, N. B. \& Krausz, E. R. Two-laser spectral hole burning in a colour centre in diamond. J. Lumin. 38, 46-47 (1987).

9. van Oort, E., Manson, N. B. \& Glasbeek, M. Optically detected spin coherence of the diamond N-V centre in its triplet ground state. J. Phys. C 21, 4385-4391 (1988).

10. Redman, D. A., Brown, S., Sands, R. H. \& Rand, S. C. Spin dynamics and electronic states of N-V centers in diamond by EPR and four-wave-mixing spectroscopy. Phys. Rev. Lett. 67, $3420-3423$ (1991).

11. Loubser, J. H. N. \& van Wyk, J. A. Electron spin resonance in the study of diamond. Rep. Prog. Phys. 41, 1201-1248 (1978).

12. Redman, D., Brown, S. \& Rand, S. C. Origin of persistent hole burning of N-V centers in diamond. J. Opt. Soc. Am. B 9, 768-774 (1992).

13. Manson, N. B. \& Wei, C. Transient hole-burning in N-V centre in diamond. J. Lumin. 58, 158-160 (1994).

14. Lenef, A. et al. Electronic structure of the N-V center in diamond: experiments. Phys. Rev. B 53, 13427-13440 (1996).

15. Martin, J. P. D. Fine structure of excited ${ }^{3} \mathrm{E}$ state in nitrogen-vacancy centre of diamond. J. Lumin. 81, 237-247 (1999)

16. Harrison, J., Sellars, M. J. \& Manson, N. B. Optical spin polarization of the N-V centre in diamond. J. Lumin. 107, 245-248 (2004).

17. Nizovtsev, A. P. et al. NV centers in diamond: spin-selective photokinetics, optical ground state spin alignment and hole burning. Physica B 340-342, 106-110 (2003).

18. Kaiser, W. \& Bond, W. L. Nitrogen, a major impurity in common type I diamond. Phys. Rev. 115 857-863 (1959).

19. Hanbury Brown, R. \& Twiss, R. Q. Correlation between photons in two coherent beams of light. Nature 177, 27-29 (1956).

20. Kurtsiefer, C., Mayer, S., Zarda, P. \& Weinfurter, H. Stable solid-state source of single photons. Phys. Rev. Lett. 85, 290-293 (2000).

21. Beveratos, A., Brouri, R., Poizat, J.-P. \& Grangier, P. in Quantum Communication, Computing and Measurement 3 (eds Tombesi, P. \& Hirota, O.) 261-267 (Kluwer Academic/Plenum, New York, 2001).

22. van Oort, E. \& Glasbeek, M. Fluorescence detected level-anticrossing and spin coherence of a localized triplet state in diamond. Chem. Phys. 152, 365-373 (1991).

23. Martin, J. P. D. et al. Spectral hole burning and Raman heterodyne signals associated with an avoided crossing in the NV centre in diamond. J. Lumin. 86, 355-362 (2000).

24. He, X. -F., Manson, N. B. \& Fisk, P. T. H. Paramagnetic resonance of photoexcited N-V defects in diamond. I. Level anticrossing in the ${ }^{3} \mathrm{~A}$ ground state. Phys. Rev. B 47, 8809-8815 (1993).

25. Smith, W. V., Sorokin, P. P., Gelles, I. L. \& Lasher, G. J. Electron spin resonance of nitrogen donors in diamond. Phys. Rev. 115, 1546-1552 (1959).

26. Holliday, K., Manson, N. B., Glasbeek, M. \& van Oort, E. Optical hole-bleaching by level anti-crossing and cross relaxation in the N-V centre in diamond. J. Phys. C 1, 7093-7102 (1989).

27. van Oort, E. \& Glasbeek, M. Cross-relaxation dynamics of optically excited N-V centers in diamond. Phys. Rev. B 40, 6509-6517 (1989).

28. van Oort, E., Stroomer, P. \& Glasbeek, M. Low-field optically detected magnetic resonance of a coupled triplet-doublet defect pair in diamond. Phys. Rev. B 42, 8605-8608 (1990).

29. Farrer, R. G. On the substitutional nitrogen donor in diamond. Solid State Commun. 7, 685-688 (1969).

30. Meijer, J. et al. Generation of single colour centers by focussed nitrogen implantation. Preprint at http://arxiv.org/abs/cond-mat/0505063 (2005)

\section{Acknowledgements}

We thank O. Gywat for valuable discussions and G. C. Farlow for high-energy electron irradiation of several samples. This work was supported by AFOSR, DARPA/MARCO and ARO.

Correspondence and requests for materials should be addressed to D.D.A.

\section{Competing financial interests}

The authors declare that they have no competing financial interests. 